\title{
OUTSOURCING NA INDÚSTRIA PARANAENSE: UM ESTUDO EMPÍRICO
}

OUTSOURCING IN PARANÁ'S INDUSTRY: AN EMPIRICAL STUDY

\author{
EDENISE APARECIDA DOS ANJOS ${ }^{1}$ \\ IVANILDO VIANA MOURA ${ }^{2}$ \\ ADRIELY CAMPAROTO BRITO3 \\ BIANCA AQUINO 4 \\ LAURO BRITO DE ALMEIDA ${ }^{5}$ \\ VICENTE PACHECO ${ }^{6}$
}

RESUMO: O outsourcing é considerado uma prática chave na organizações industriais e prestadoras de serviços, consiste na tomada de decisão estratégica entre produzir internamente produtos ou serviços ou comprar de fontes externas, objetivando a redução de custos e melhor desempenho. No entanto, antes de decidir pela aderência à prática, os gestores se veem diante de fatores que pesam na decisão, os quais demandam análise principalmente do impacto nas atividades operacionais da organização. Nesse contexto, a pesquisa objetivou investigar quais os critérios recorrentes para a tomada de decisão na adesão do outsourcing, na indústria paranaense sob a percepção dos gestores. Quanto aos delineamentos metodológicos a pesquisa é classificada como descritiva com enfoque quantitativo, a coleta de dados se deu por meio de questionário adaptado de Moschuris (2015), nos meses de junho e julho de 2016, a população e amostra foram extraídas do banco de dados da Federação das Indústrias do Paraná (FIEP), como amostra final foram analisadas 33 indústrias respondentes. Os resultados obtidos evidenciaram que dentre os 11 critérios estratégicos abordados, os itens de custos e qualidade foram considerados como mais relevantes na percepção dos gestores respondentes para tomada de decisão de adesão ou não ao outsourcing. Quanto as dimensões em que as decisões são tomadas, a mais importante foi considerada pelos gestores, foi a da complexidade, seguida de tecnologias e incertezas ambientais.

Palavras-chave: Outsourcing. Produzir. Comprar. Redução de custos. Tomada de decisão.

ABSTRACT: Outsourcing is considered a key practice in industrial organizations and service providers. It consists of making a strategic decision between producing products or services internally or buying from external sources, with the aim of reducing costs and improving performance. However, before deciding to adhere to the practice, managers are faced with factors that weigh in the decision, which demand analysis mainly of the impact on the

Data de submissão: 16/01/2018. Data de aceite: 14/12/2018. Data de publicação: 17/12/2018.

\footnotetext{
${ }^{1}$ Mestre em Contabilidade e Finanças - Universidade Federal do Paraná (UFPR, 2015-2017). Docente na Universidade Federal de Santa Catarina.

2 Mestre em Contabilidade pela Universidade Federal do Paraná - UFPR (2015-2017). Professor no Centro Universitário Internacional - UNINTER.

${ }^{3}$ Mestre em Contabilidade pela Universidade Federal do Paraná - UFPR. Possui experiência em contabilidade comercial, tributária, contabilidade de custos e gerencial. Interesse de pesquisa em Controle Gerencial.

${ }^{4}$ Mestre em Contabilidade pela Universidade Federal do Paraná - UFPR (2015-2016).

5 Doutor em Controladoria e Contabilidade pela Universidade de São Paulo (1999) e Pós-Doutorando em Controladoria e Contabilidade EAC|FEA|USP. Professor Associado III na Universidade Federal do Paraná. Endereço: Avenida Prefeito Lothário Meissner, 632 - Jardim Botânico, Setor de Ciências Sociais Aplicadas. CEP: 80.210-170 - Curitiba-PR. Email: laurobrito52@gmail.com

${ }^{6}$ Doutor em Engenharia de Produção pela Universidade Federal de Santa Catarina - UFSC (2004). Professor Associado III na Universidade Federal do Paraná - PPGCONT.
} 
operational activities of the organization. In this context, the research aimed to investigate the recurrent criteria for decision making in outsourcing adherence, in the industry of Paraná under the perception of the managers. As for the methodological delineations the research is classified as descriptive with a quantitative approach, the data collection was done through a questionnaire adapted from Moschuris (2015), in the months of June and July of 2016, the population and sample were extracted from the database of the Federation of Industries of Paraná (FIEP), as final sample were analyzed 33 respondent industries. The results obtained evidenced that among the 11 strategic criteria addressed, the cost and quality items were considered as more relevant in the perception of the respondent managers for decisionmaking to join or not to outsourcing. As far as the dimensions in which the decisions are taken, the most important was considered by the managers, was the complexity, followed by technologies and environmental uncertainties.

Keywords: Outsourcing. Produce. Purchase. Reduced costs. Decision making.

\section{INTRODUÇÃO}

No atual cenário competitivo vivenciado pelas empresas, todas as estratégias conhecidas valem como diferencial no que tange à redução de custos, visando diminuir o preço de produtos/serviços e posicionar-se à frente da concorrência. Nesse contexto, uma prática que vem ganhando destaque é o outsourcing, que conforme Leeman e Reynolds (2012) consiste na utilização de serviços de terceiros para realizar funções que normalmente têm sido realizados internamente.

O outsourcing possibilita às empresas concentrar seus recursos em suas competências essenciais, reduzindo custos e aumentando a flexibilidade por meio de parcerias ao longo da cadeia de suprimentos (LEE, et al. 2018). Para Aubert, Kishore, e Iriyama (2015) a prática de outsourcing inserida nas organizações encaixa-se no meio de um sistema dinâmico com a participação de fornecedores, clientes e concorrência, contexto no qual a competência dos parceiros faz com que seus limites tornem-se permeáveis, abrindo novas possibilidades no mercado.

No entanto, a decisão de adesão ao outsourcing, mesmo na expectativa de benefícios diversos, deve ser muito bem avaliada pela administração, que deve conhecer e considerar todos os fatores que venham a impactar na estrutura organizacional da entidade. Conforme Zhu (2016) na terceirização a empresa fica dependente de terceiros para operacionalizar parte de suas atividades, e caso não sejam adequadamente gerenciadas, poderá ter seu desempenho negativamente afetado. Nesse sentido, El Mokrini, Dafaoui, Berrado, El Mhamedi (2016) enfatizam que as decisões em torno do outsourcing também lidam com a avaliação e identificação de riscos operacionais e estratégicos, o que pode levar a necessidade de diferentes habilidades gerenciais por parte dos gestores.

Embora os motivos mais comuns observados para a utilização da prática de outsourcing sejam os benefícios com a redução de custos devido à economia superior dos fornecedores de escala e/ou salários mais baixos (BENGTSSON; HAARTMAN; DABHILKAR, 2009), existem outros fatores que podem influenciar a tomada de decisão com relação à adoção ou não à sua adesão. Um dos pressupostos segundo Kakabadse e Kakabadse (2005) é de que um único fornecedor não possui recursos de classe global para todas as áreas do negócio, implicando a necessidade de parceria com outros fornecedores. Para EspinoRodriguez e Padron-Robaina (2006) tradicionalmente, os estudos sobre outsourcing e seus determinantes focam abordagens econômicas, que consideradas isoladamente, fazem com que sejam ignorados outros aspectos do comportamento organizacional, os quais podem ter 
impactos importantes sobre as respectivas decisões.

Neste contexto, a questão norteadora dessa investigação consiste em investigar: quais os critérios recorrentes para a tomada de decisão na adesão do outsourcing? Deste modo, busca-se investigar na percepção dos gestores, os critérios e as variáveis considerados relevantes para tomada de decisão de adesão ao outsourcing. Considerando a escassez de pesquisa sobre o tema esse artigo tem como objetivo agregar informações sobre a temática de outsourcing, verificando essa prática de tomada de decisões nas indústrias paranaenses de exportações e importações, bem como analisar quais são os critérios com maior impacto nessa questão específica.

O outsourcing difunde-se cada vez mais entre organizações sendo uma das decisões estratégicas que atraem o maior interesse de profissionais e estudiosos organizacionais (ESPINO-RODRIGUEZ; PADRON-ROBAINA, 2006). Justifica-se a presente investigação pelo fato de abrir novos caminhos para as pesquisas em outsourcing, bem como contribuir para a literatura contábil, voltada ao tema em empresas paranaenses e o impacto das variáveis como tipo e complexidade intrínsecas ao produto ou serviço e relativo a incerteza ambiental e a tecnologia disponível envolvendo questões táticas.

Na próxima seção é abordada a literatura relevante com o marco teórico-empírico, seguido pela metodologia trazendo o modelo e hipóteses de pesquisa, finalizando com os resultados e conclusões, apresentando sua contribuição tanto para a compreensão acadêmica como para a melhoria da prática mercadológica. Também é apresentado limitações desse estudo e oportunidades para futuras pesquisas.

\section{REFERENCIAL TEÓRICO}

Neste tópico, serão apresentados os temas decorrentes dos assuntos abordados na pesquisa, os quais dizem respeito basicamente à conceituação do outsorcing, bem como fatores e critérios que impactam na decisão do mesmo.

\subsection{Make-or-Buy}

Durante a década de 1980, a crescente instabilidade dos mercados e o aumento da competitividade entre as empresas, levaram-nas a recorrer à terceirização de certas atividades, quebrando com o paradigma da produção interna de todas as partes dos seus processos produtivos (GUIMARÃES, 2017). Com o advento da globalização, a busca por menores custos e maior competitividade fez com que essa prática se acentuasse e, de acordo com Leeman e Reynolds (2012) estima-se que a terceirização de bens e serviços no mundo inteiro continua a crescer.

O outsorcing está relacionado às estratégias de redução de custos, envolvendo atividades para as quais a empresa não possui necessidades críticas nem capacidades especiais (BAATARTOGTOKH; DUNBAR; ZYL, 2018). Diante disso, o termo outsorcing é hoje empregado para a compra de produtos e de serviços externos e, de acordo com Guimarães (2017), esse recurso nem sempre traz benefícios, sendo então importante analisar as razões que levam ao make-or-buy (fazer ou comprar), ou seja, decidir se a empresa realiza todas as atividades relacionadas ao seu processo produtivo ou se compra parte de fornecedores externos.

A prática do outsorcing está geralmente relacionada com funções que estão fora da área de atuação da empresa, permitindo que ela se concentre em suas principais competências, e deixando as atividades secundárias por conta de um fornecedor externo (EL MOKRINI; et al., 2016). Nesse contexto, Mclvor (2009) afirma que a lógica do outsorcing está 
relacionada ao fato de que as organizações devem realizar internamente as atividades que Ihes são valiosas, raras e difíceis de imitar, ganhando com isso, vantagem competitiva.

As decisões de nível estratégico permitem às empresas comprarem de outras empresas itens os quais não oferecem vantagem operacional para a linha de produção interna, independente do motivo que leve a essa decisão a intenção é melhorar os resultados aumentando a lucratividade, não apenas considerando aspectos econômicos, mas agregando nessa decisão situação da posição da empresa, capital de giro, posição competitiva entre outros (BAJEC; JACOMIN, 2010).

Para fazer face à concorrência, as empresas devem ser eficientes no fornecimento de produtos ao mercado em tempo hábil e dentro das possibilidades do seu orçamento, levando em conta também que as exigências e as dinâmicas do ambiente de inserção, o que as impulsionam a aderir à técnica de outsourcing, na qual transferem a terceiros a responsabilidade de ter especialistas, instalações e equipamentos (TROACĂ; BODISLAV, 2012).

A opção make or buy não é uma decisão simples. O trabalho de projetar, administrar, produzir e refinar continuamente produtos ou serviços consome tempo, habilidades e é dispendioso (FIGUEIREDO; BREMER; MALDONADO, 2003). Neste sentido, Zhu (2016), destaca que o outsorcing é uma estratégia que permite a empresa focar em suas competências essenciais, pois caso ela não possua habilidades ou conhecimentos específicos para um determinado trabalho, a terceirização passa a ser uma das melhores opções.

Nesse entendimento, Tavana et al., (2016), afirmam que dentre as decisões acerca da opção de make or buy, um dos fatores mais importantes a ser considerado é a identificação de quais atividades devem ser realizadas internamente e quais devem ser terceirizadas. Diante desse aspecto, torna-se necessário conhecer todas as atividades envolvidas no processo de produção de um produto ou serviço, para então analisá-las e fazer a identificação das que mais agregam valor ao negócio.

Outro aspecto importante de ser destacado é em relação às limitações de recursos das empresas, pois conforme Tavana et al., (2016) desenvolver as habilidades necessárias para cumprir tarefas específicas por meio de treinamentos constantes pode ser muito demorado, exige muito esforço e pode sair muito caro. Portanto em algum momento a empresa terá que decidir entre make or buy buscando manter-se no mercado sem precisar necessariamente de vultuosos investimentos.

As estratégias operacionais e táticas passam por essa decisão de fazer na estrutura operacional da empresa ou externalizar o processo (BAJEC; JACOMIN, 2010). Essa decisão permite a empresa investir seus recursos, sejam eles materiais ou humanos, em operações mais vantajosas para a linha de produção, tornando-se uma tarefa importante da gestão empresarial e um item importante, pois uma decisão equivocada pode levar a insucesso empresarial (BAJEC; JACOMIN, 2010).

Diversos podem ser os critérios que influenciam as decisões pelo make or buy. De acordo com El Mokrini et al., (2016) atualmente as empresa estão conscientes das vantagens pela terceirização, as quais podem incluir a redução de custos operacionais, a troca de custos fixos por custos variáveis, eliminação de investimentos em infra estrutura, respostas mais rápidas às mudanças no ambiente dos negócios, compartilhamento de riscos, acesso à recursos limitados, etc. A complexidade de uma apuração completa sob make-or-buy é devido à dinâmica e incertezas das atividades empresariais. Múltiplos fatores têm implicações para essa decisão. Algumas abordagens úteis na compreensão da adoção de práticas de outsourcing no mercado empresarial podem ser colocadas numa visão baseada em três 
pilares: (i) quanto a perspectiva das dimensões da incerteza ambiental, (ii) quanto aos tipos de tecnologias e, (iii) quanto as dimensões da complexidade (MOSCHURIS, 2015).

\subsection{Critérios estratégicos para tomada de decisão em outsourcing}

A tomada de decisão por outsourcing, tornou-se uma prática imperativa nas organizações, uma estratégia chave na indústria e alguns setores de serviços (GUNASEKARAN et al., 2015). Isto posto, as organizações se valem das práticas de outsourcing na produção de produtos e serviços de externamente, para obter vantagem competitiva, focando em forças e flexibilidade, segurança da informação, localização geográfica, entre outros fatores inerentes de cada segmento de atuação (PRATAP, 2014; GERBL, et al., 2015; GUNASEKARAN et al., 2015; BALS; TURKULAINEN, 2017). No entanto, as organizações utilizam estrategicamente o outsourcing visando essencialmente redução de custos e melhor desempenho.

Sob esta ótica, o outsourcing das atividades de produção tem apresentado um efeito significativo sobre a forma como as indústrias de transformação e serviços têm desenvolvido seus produtos, no que tange as decisões make or buy, ou seja, produzir internamente, ou comprar de um fornecedor externo (MEIXELL; KENYON; WESTFALL, 2014). De modo que as atividades que não são competências essenciais, devem ser transferidas para terceiros, permitindo à entidade concentrar-se naquilo que ela consegue fazer com maior qualidade, produtividade e menor custo (FERRUZZI, et al. 2011; MEIXELL, 2014).

Observa-se, no entanto, que a prática do outsourcing tem sido utilizada no decorrer do tempo de várias formas, com objetivos distintos, os quais se iniciaram com foco em redução de custos e posteriormente tornaram-se estratégias organizacionais. Entretanto, esta é uma decisão potencialmente complexa, pois geralmente envolve questões estratégicas e operacionais, possivelmente com impacto significativo no desempenho financeiro (NIELSEN; MITCHELL; NØRREKLIT, 2015). Desse modo, a decisão por práticas de outsourcing, devem considerar além dos fatores econômicos, fatores operacionais tais como produção, mão-obra, recursos materiais, recursos humanos, logística, complexidades dos processos, tecnologias, além de aspectos do comportamento organizacional que podem ter efeitos substanciais sob a decisão da adesão (MOSCHURIS, 2015).

As dimensões da estratégia de outsourcing emergem das decisões conscientes e inconscientes das organizações ao utilizar fontes externas para produção de sua atividade principal, envolvem várias dimensões do desempenho organizacional, incluindo inovação, qualidade, lucratividade, receita e eficiência em custos (MAGELSSEN; SANCHEZ; DAMANPOUR, 2015). Diante disso, Moschuris (2015) evidencia outras variáveis que podem influenciar os critérios para tomada de decisão, relacionadas com incertezas ambientais, complexidade do produto/serviço e tipos de tecnologias.

\subsubsection{Dimensões da Incerteza Ambiental}

Abordar desenvolvimento de novos produtos, mudanças tecnológicas na empresa e no mercado, lançamentos de novos produtos, alterações nas especificações, entre outros aspectos envolvidos no ambiente mercadológico requer adotar um comportamento diferenciado na tomada de decisões. Premissa que resulta maior dificuldade administrativa em ambientes complexos (MOSCHURIS, 2007). Com essas considerações, tem-se que ambientes complexos comportam mudanças rápidas e exigem mais das empresas a necessidade de manter as decisões em níveis mais elevados da organização (MOSCHURIS, 2007). 
Em ambientes de alto grau de incertezas ambientais espera-se que as empresas sejam mais propensas a reter os processos internamente, no entanto Gerbl et al., (2015) observaram que em alguns casos a incerteza ambiental atua como agente motivadora de práticas de outsourcing, principalmente no que tange a flexibilidade em arranjos dos fornecedores externos, para permitir mudanças na escala de operações, desenvolver novas capacidades de acordo com as necessidades.

Outro fator a ser considerado é o risco que se corre ao aderir à prática de outsorcing. Conforme exposto por El Mokrini et al. (2016), na fase de terceirização lida-se com a identificação e avaliação dos riscos potenciais, podendo os mesmos ser classificados como operacionais e estratégicos. Dentre os riscos que podem ocorrer nesse processo, pode-se citar a capacidade de coordenar com o fornecedor, manutenção do cronograma de produção e controle de custos (BAATARTOGTOKH; DUNBAR; ZYL, 2018). Dessa forma, tanto as incertezas ambientais quanto os riscos devem ser analisados quando da proposta de aderência ao outsourcing, pois esses fatores podem impactar no desempenho organizacional.

\subsubsection{Dimensões de Complexidade - tipo de produto e serviço}

As Dimensões de Complexidade e/ou tipo de produto e serviço também são aspectos que impactam na decisão de adesão ao outsourcing. Entre as questões estratégicas de decisões nas empresas e determinantes de rentabilidade permeiam o grau de diferenciação, complexidade técnica, instalação especializada e existência de técnicos de assistência pós venda (MOSCHURIS, 2007).

Nesse sentido, Moschuris (2007) coloca que em muitas organizações as decisões de outsourcing já passam por setores de marketing, contabilidade, jurídico, controle de qualidade, limpeza, segurança e manutenção, e na visão de Moschuris (2015), o aumento da complexidade gera conhecimento sobre o desenho do modelo limitado da empresa onde a mesma se vê diante da opção de transferir essas responsabilidades para terceiros, podendo se concentrar em suas atividades de competência.

\subsubsection{Tipos de Tecnologia de Operações}

Nos últimos anos houve o incremento significativo no setor de outsourcing o que foi eficaz para a geração interna de conhecimento e inovação. As empresas estão cada vez mais em uma busca por soluções e por vezes aderem às parcerias. Essa inovação requer um desenvolvimento contínuo com o intuito de melhorar a oferta de produtos ou serviços da empresa, ou permitir novos mercados (OSHRI; KOTLARSKY; GERBASI, 2015).

As diretrizes para determinar as direções apropriadas para um arranjo empresarial demandam uma estruturação dessa cadeia logística atrelada com o programa estratégico que retrate o cenário organizacional, sendo que a definição de táticas define o caminho da empresa sobrevindo uma captação das atividades a serem tomadas pelo novo plano, para os intrincados organizacionalmente (CARVALHO, 2012; GANZER, et al. 2013).

Estudos evidenciam que a característica organizacional da empresa considerando tecnologia de operações pode influenciar o sistema de decisões entre fazer e comprar, porém esse papel da tecnologia de operações ainda é controverso entre as organizações (MOSCHURIS, 2007). Interferindo na saúde financeira da empresa, uma das competências da organização é mesclar tecnologias com habilidades de produção, determinando a vantagem competitiva. Essa estratégia é muito dinâmica e flexível e deve evoluir conforme a tecnologia disponível (MOSCHURIS, 2008). 
Em síntese, o ponto chave do outsourcing, reside nas transferências estratégicas das necessidades e processos da empresa aos fornecedores, que estão melhores colocados para executar e melhorar na área específica da atividade. Fornecem recursos tecnológicos de ponta no seu domínio de especialização e relativamente livre de forças burocráticas, esses fornecedores oferecem acesso a suas capacidades superiores como também os ganhos de futuras inovações para as empresas clientes (PRATAP, 2014).

\section{DELINEAMENTOS METOdOLÓGICOS}

A pesquisa empírica realizada é descritiva quanto ao objetivo proposto por apresentar características de um determinado fenômeno, sem influenciar ou interferir no mesmo (COOPER; SCHINDLER, 2003), com enfoque quantitativo quanto à abordagem do problema, por confiar na medição numérica e uso da estatística para estabelecer os padrões de comportamento do objeto de estudo (SAMPIERI; COLLADO; LUCIO, 2013).

$O$ instrumento de pesquisa utilizado para coletar os dados neste estudo foi traduzido e adaptado de Moschuris (2015). A adaptação foi realizada após a tradução, considerando o contexto nacional, ajustando vocabulário e expressões para a língua nacional, retratando o mercado local. Após a adaptação, foi realizado um pré-teste com os alunos do programa de mestrado e doutorado em Contabilidade, da Universidade Federal do Paraná, a fim de avaliar a adaptação feita. O questionário foi estruturado em dois blocos, sendo o primeiro abrangendo 5 (cinco) questões fechadas para obter informações a respeito dos critérios e dimensões utilizados para tomada de decisão de adesão ao outsourcing e 1 (uma) questão aberta para indicar o item ou serviço para o qual for adotado o outsourcing nos últimos 6 (seis) meses. Nas questões fechadas do primeiro bloco utilizou-se a escala de mensuração do tipo Likert de 5 (cinco) pontos, sendo as opções entre 1 (um) para muito baixa e 5 (cinco) para muito alta. No segundo bloco foram apresentadas 7 (sete) questões abertas e 2 (duas) fechadas para obtenção de informações a respeito do perfil da empresa e do respondente.

A população objeto deste estudo é formada por 1056 indústrias de transformação, atuantes no Estado do Paraná, com cadastro ativo junto a Federação das Indústrias do Paraná (FIEP), conforme banco de dados disponibilizado. Com vistas a delimitar a população, foram selecionadas as indústrias que atuam com exportações e importações, devido as variáveis ambientais e mercadológicas a que estão expostas. A amostra utilizada pode ser definida como uma amostra não-probabilística, censitária por adesão. Inicialmente formada por 48 indústrias das quais foram recebidos questionários devidamente respondidos. No entanto, no questionário a primeira questão inserida, tinha como objetivo, selecionar apenas as indústrias que utilizaram práticas de outsourcing nos últimos 6 meses, excluindo-se dessa forma 15 respondentes da amostra, desse modo a amostra final corresponde à 33 indústrias respondentes.

A coleta de dados ocorreu nos meses de junho e julho de 2016. Para coletar os dados relativos à percepção dos respondentes disponibilizou-se um link para acesso ao questionário elaborado na plataforma Google Docs ${ }^{\circledR}$ encaminhado no e-mail constante no banco de dados de cadastro das indústrias disponibilizados pela FIEP, endereçados preferencialmente ao gerente geral da indústria, para que este fosse o respondente e, em sua ausência, alguém de cargo semelhante que participasse do processo de tomada de decisões a respeito de produzir ou comprar itens ou serviços do processo produtivo organizacional. Destaca-se que foram enviados e-mails mais de uma vez para a população total, na tentativa de ampliar o índice de respondentes e por fim foram realizados contatos via telefone. 
Para tratamento dos dados utilizou-se o Software Statistical Package for Social Sciences - SPSS 18.0 de onde foi possível extrair os dados de estatística descritiva utilizados na pesquisa.

\section{ANÁLISE DOS DADOS}

Das 33 respostas válidas, observou-se que os respondentes estão estabelecidos em diversas regiões geográficas do Estado do Paraná. Quanto ao segmento de atuação da indústria, $27 \%$ é composto por indústrias metalúrgicas, $6 \%$ indústria alimentícia, $6 \%$ indústria de equipamentos elétricos, $6 \%$ indústria de equipamentos de eletromecânica, $40 \%$ da amostra ficou distribuída entre as indústrias química, de equipamentos de aquecimento, madeireira, de equipamentos agrícolas, de rações para animais, de vestuário, de carrocerias, de plásticos e de móveis e $15 \%$ não identificaram o segmento de atuação da indústria.

Em relação ao número de colaboradores diretos, 49\% da amostra possui entre 20 e 99 colaboradores, $42 \%$ possui entre 100 e 499 colaboradores e $9 \%$ possui até 19 colaboradores, indicando que $91 \%$ da amostra possui mais de 20 colaboradores diretos, podendo ser consideradas de pequeno e médio porte.

Figura 1-Dashboard dados da pesquisa

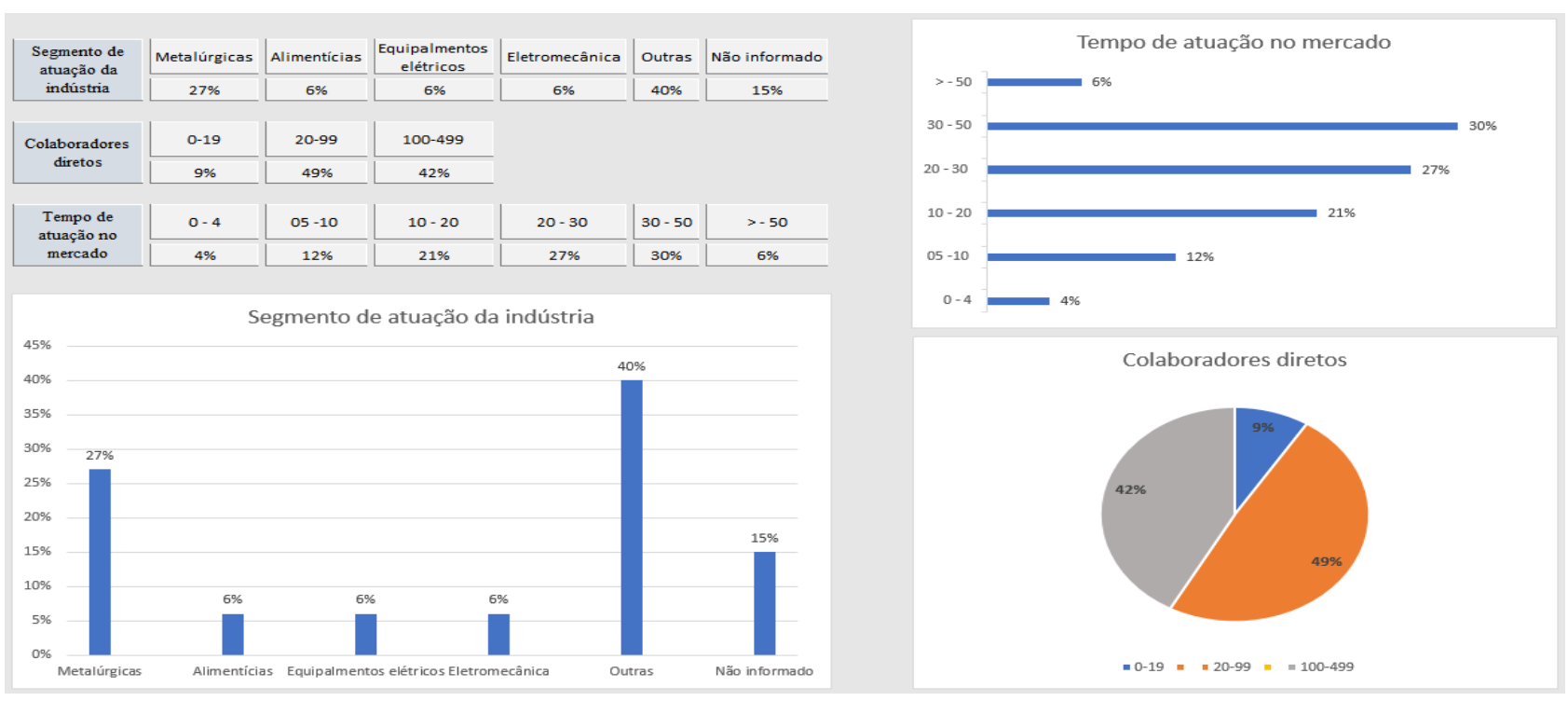

Fonte: Dados da pesquisa.

Analisando-se o tempo de atuação no mercado, 30\% da amostra possui entre 30 e 50 anos, $27 \%$ possui entre 20 e 30 anos, 21\% possui entre 10 e 20 anos, $12 \%$ possui entre 5 e 10 anos, $6 \%$ possui mais de 50 anos e $4 \%$ possui menos de 5 anos, apontando que $61 \%$ da amostra é composta por indústrias com mais de 20 anos de atuação.

O perfil dos respondentes é caracterizado por 8 sócios proprietários ou diretor, 8 gerentes de produção, 8 gerentes de compras e comercial, 4 gerentes gerais, e 5 gerentes de outros setores da indústria como contabilidade, marketing, supervisão, financeiro e custo e recursos humanos. Quanto a tempo de experiência apresentado 11 possuem menos de 5 anos, 10 possuem entre 5 e 10 anos, 7 possuem entre 10 e 20 anos e 5 possuem mais de 20 anos de experiência, indicando que mais da metade dos respondentes possuem menos de 10 anos de experiência profissional como tomador de decisões.

Quanto item ou serviço para o qual as indústrias em que os respondentes atuam, tenham tomado a decisão de comprar ou terceirizar ou invés de produzir internamente nos 
últimos 6 meses, 31\% aderiram para fabricação de peças, 25\% para serviços auxiliares do processo produtivo, $15 \%$ para fabricação de unidades de produção, $15 \%$ para aquisição de insumos, $8 \%$ para tecnologias, 3\% para mão de obra especializada e $3 \%$ para serviços de transportes. Corroborando com Figueiredo, Bremer e Maldonado (2003) ao abordarem que o outsourcing abrange tantos produtos físicos como serviços, para os quais o projeto, a administração e o refinamento consomem tempo, habilidades e é dispendioso.

O estudo que fez despertar o interesse por esta investigação é Moschuris (2015) que pesquisou os critérios que devem ser considerados na resolução tática de decisão de 'make or buy' na Grécia, para tanto dividiu a pesquisa em duas etapas. A primeira realizou entrevista com gestores de 10 indústrias, dessa analise resultaram 11 critérios considerados relevantes para tomada de decisão do outsourcing, sendo eles: custo, qualidade, tempo, carga de trabalho, experiência, situação financeira, projeto sigiloso, controle de produção, controle de distribuição, proteção ambiental e legislação.

Com a análise das entrevistas, tinham-se bastante detalhamento, entretanto, não se pode generalizar esses resultados. Para tanto, o autor desenvolveu a segunda etapa da pesquisa, com a elaboração de um questionário que permitisse identificar a relevância desses 11 critérios apontados anteriormente, juntamente com as dimensões que podem influenciar também na tomada de decisão de adesão ou não ao outsourcing e, para validação do mesmo, solicitou a um grupo de pesquisadores acadêmicos especializados no campo das compras, um tipo de consulta aos especialistas, para que fizessem as contribuições necessárias (MOSCHURIS, 2015).

Com as adaptações feitas, realizou um pré-teste com 50 empresas industriais não incluídas na amostra final utilizada para análise. Esses procedimentos implicam em elevado grau de validade do conteúdo do questionário, que foi direcionado aos gerentes gerais e então aplicado a uma população de 300 empresas industriais gregas que possuíam mais de 100 colaboradores diretos, selecionadas aleatoriamente. Ao final da coleta de dados obtiveram 85 respostas validas, ou seja, um retorno de $28,3 \%$ que foram tratadas estatisticamente e apresentaram os resultados explorados a seguir (MOSCHURIS, 2015).

Considerando os procedimentos realizados pelo pesquisador para elaboração e validação do questionário, a relevância do tema e a pouca quantidade de pesquisas brasileiras relacionadas ao assunto, justificam a escolha do instrumento de pesquisa de Moschuris (2015) como norteador para esta investigação, podendo-se dessa forma, proporcionar às empresas brasileiras uma relação de critérios que devem ser considerados na tomada de decisão de produzir ou comprar (make or buy).

Assim como no estudo original, a população é composta somente por indústrias, todavia não se limitou a amostra conforme a quantidade de colaboradores. Moschuris (2015) desenvolveu a pesquisa na Grécia, e essa pesquisa abrangeu somente o Estado do Paraná por ser um dos grandes centros industriais brasileiros e estado de localização atual dos pesquisadores. Quanto aos segmentos participantes da amostra, em ambos estudos foram diversificados e participaram indústrias alimentícias, de vestuário, de plásticos, de produtos químicos, metalúrgicas entre outros, destacando-se como maior proporção da amostra a indústria alimentícia grega e a metalúrgica paranaense.

Conforme apresenta Vernalha e Pires (2005), a decisão de adesão ao outsourcing é utilizada como uma estratégia das organizações que possibilita repassar a terceiros serviços ou etapas do processo produtivo, enquanto concentram-se esforços e recursos nas principais competências desempenhadas, o que exige habilidades gerenciais dos gestores de modo que consigam integrar e administrar todas as atividades desenvolvidas. Todavia essa decisão 
precisa ser subsidiada com informações a respeito dos fatores e impactos que podem ocorrer na estrutura organizacional, pois o desempenho pode estar sujeito também às variáveis tais como uma crise financeira e interrupção na cadeia de suprimentos (BALS; KIRCHOFF; FOERSTL, 2016). Bajec e Jacomin (2010) explicam que fazer a escolha certa e desenvolver uma gestão bem-sucedida sustenta uma vantagem competitiva. Nesse sentido os resultados desta pesquisa trazem contribuições aos gestores ao apresentar os fatores considerados por organizações que já aderiram ao outsourcing.

Dos 11 critérios estratégicos apresentados no questionário pode-se observar que as variáveis consideradas mais relevantes na percepção dos gestores respondentes desta pesquisa foram custo e qualidade, semelhante ao resultado obtido por Moschuris (2015) conforme apresentado na Tabela 1, que demonstra os itens de critérios relevantes para a tomada de decisão de adesão ou outsourcing por ordem decrescente de média apresentada.

Tabela 1 - Critérios estratégicos para decisão de adesão ao make or buy

\begin{tabular}{llllll}
\hline Pesquisa atual & \multicolumn{5}{l}{ Pesquisa Moschuris (2015) } \\
\hline Critérios & Média & Desvio Padrão & Critérios & Média & Desvio Padrão \\
\hline Custos & 4,09 & 0,94 & Custo & 4,21 & 0,71 \\
Qualidade & 3,64 & 1,22 & Qualidade & 4,06 & 1,23 \\
Experiência & 3,42 & 1,43 & Tempo & 3,71 & 1,17 \\
Tempo & 3,24 & 1,32 & Experiência & 3,24 & 1,56 \\
Carga de trabalho & 3,03 & 1,31 & Posição financeira & 2,31 & 1,45 \\
Controle de distribuição & 2,82 & 1,31 & Workload & 2,23 & 1,45 \\
Posição financeira & 2,64 & 1,45 & Projeto sigilo & 2,00 & 0,96 \\
Controle de produção & 2,55 & 1,27 & Controle de produção & 1,61 & 1,01 \\
Legislação & 2,12 & 1,38 & Proteção ambiental & 1,60 & 1,07 \\
Proteção Ambiental & 1,85 & 1,25 & Legislação & 1,45 & 0,99 \\
Projeto sigiloso & 1,67 & 0,95 & Controle de distribuição & 1,40 & 0,89
\end{tabular}

Fonte: dados da pesquisa.

Nota-se que em ambas as pesquisas o critério mais relevante considerado pelos gestores é a variável custos, corroborando alguns teóricos ao ressaltarem que apesar da importância de decisões a respeito de quais etapas do processo produtivo ou os serviços que podem ser adquiridos de terceiros, muitas vezes estas decisões são baseadas em perspectivas de curto prazo e de custos (BAJEC; JACOMIN, 2010; BALS; KIRCHOFF; FOERSTL, 2016; MCIVOR, 2013). A busca pela redução de custos destaca-se como um dos principais motivadores nestas situações, almejando a melhoria do desempenho do negócio, visto que a organização se dedica em atividades das quais consegue fazer com maior qualidade, produtividade e menor custo, transferindo para terceiros aquelas atividades que não configuram sua competência essencial (BALS; KIRCHOFF; FOERSTL, 2016; ZHU, 2016; LEE, et al. 2018; BAATARTOGTOKH; DUNBAR; ZYL, 2018; FERRUZZI, et al. 2011).

A utilização de outsourcing no decorrer do tempo evoluiu evidenciando objetivos distintos. No início o foco é a redução de custos e posteriormente essa técnica tornou-se uma poderosa estratégia das organizações para cumprimento de metas e objetivos e diversos contextos e, por isso considerá-la apenas em abordagem econômica (redução de custos) é desprezar os demais aspectos do comportamento organizacional. (ESPINO-RODRIGUEZ; PADRON-ROBAINA, 2006).

Nesse sentido, outra variável relevante considerada na adesão do make or buy é a qualidade, apontada como segundo maior motivador em ambas as pesquisas. A relevância desse item evidência a preocupação dos gestores em manter a qualidade dos produtos finais, uma vez que representam a fonte de recurso da organizacional e, a redução desta pode afetar o espaço da empresa no mercado reduzindo sua competitividade. 
As variáveis de experiência e tempo, apresentadas pelos gestores, se revezam em terceiro e quarto critério na ordem de relevância na pesquisa atual em comparação com a de Moschuris (2015), evidenciando a preocupação dos gestores em relação ao tempo utilizado nas etapas do processo produtivo até a entrega ao cliente, assim como a experiência da mão de obra no desenvolvimento das atividades. Esses fatores podem orientar a decisão de outsourcing e são fundamentais para manutenção da competitividade das organizações. Para Burt, Petcavage e Pinkerton (2010) e Baatartogtokh, Dunbar e Zyl (2018) os benefícios do conhecimento especializado disponível fora da organização pode conduzi-la à aquisição ao invés de produzir o item ou serviço.

Do quinto ao último item de critérios apresentados pelos respondentes da pesquisa atual se diversificaram em relação à ordem de relevância dos achados de Moschuris (2015). Destacando-se a posição financeira que é considerada o quinto item relevante para decisão de adesão ao outsourcing na Grécia e no Paraná foi apontado como sétimo critério, e o controle de distribuição apontado como sexto item na ordem de relevância pelos gestores das indústrias paranaenses enquanto que para os gregos esse é o item de menor relevância. Uma possível explicação para essa divergência pode estar relacionada com as condições logísticas brasileiras.

Moschuris (2015) explica que o baixo impacto de alguns critérios tais como controle de produção, legislação, proteção ambiental, sigilo de projetos e controle de produção pode ser justificado pela particularidade dos projetos, itens ou serviços pelos quais se optam pela aquisição de terceiros, pois em alguns casos essa decisão pode se apresentar como momentânea.

Outras variáveis além de custos e lucro, quando considerados em conjunto podem afetar significativamente a rentabilidade das organizações, como é o caso dos critérios de qualidade, prazo e desempenho na entrega, conforme apresentados por Kamble e Ghosh (2010) e Kulkarni e Jenamani (2008). Há ainda que se considerar que a dinâmica e incertezas da atividade empresarial e outros fatores podem tornar a decisão de adesão ao outsourcing mais complexas, sendo necessário compreender algumas abordagens quanto a essa perspectiva, sendo elas: a incerteza ambiental, os tipos de tecnologias e as dimensões de complexidade.

Cinco variáveis propostas por Moschuris (2015) foram utilizadas para avaliar a incerteza ambiental, sendo elas: frequência de alterações nas especificações de produtos oriundos tanto de iniciativa interna como de órgãos regulamentadores das atividades específicas, item este considerado como mais relevante pelos gestores respondentes desta pesquisa; o segundo item é a frequência de mudanças tecnológicas no mercado; o terceiro é a frequência do aparecimento de novos produtos no mercado, seguido por frequência de mudanças tecnológicas na empresa e frequência de desenvolvimentos de novos produtos na empresa. Esses elementos juntos permitem avaliar a dimensão da incerteza ambiental, conforme apresentado na Tabela 2, a média e desvio padrão dos elementos.

Tabela 2 - Avaliação da dimensão de incerteza ambiental

\begin{tabular}{lll}
\hline Dimensão de Incerteza Ambiental & Média & Desvio Padrão \\
\hline Frequência de alterações nas especificações & 2,91 & 1,33 \\
Frequência de mudanças tecnológicas no mercado & 2,76 & 1,27 \\
Frequência do aparecimento de novos produtos no mercado & 2,64 & 1,29 \\
Frequência de mudanças tecnológicas na empresa & 2,39 & 1,02 \\
Frequência de desenvolvimento de novos produtos na empresa & 2,27 & 1,15 \\
\hline
\end{tabular}

Fonte: dados da pesquisa. 
Outra dimensão que pode impactar na decisão de adesão ao outsourcing é a utilização de diversos tipos de tecnologias de operações, para mensurar esse contexto, foram utilizadas variáveis que indicam aplicação de tecnologias em produção de pequenos lotes, produção de lotes personalizados, produção de processamento contínuo, produção de grandes lotes e produção em massa, apontados nessa ordem de relevância pelos gestores respondentes desta pesquisa, apresentadas na Tabela 3.

Tabela 3 - Avaliação da dimensão de tipos de tecnologias de operações

\begin{tabular}{lll}
\hline Tipos de Tecnologia de Operações & Média & Desvio Padrão \\
\hline Lote pequeno & 3,48 & 1,39 \\
Personalizado & 3,18 & 1,40 \\
Processamento contínuo & 3,03 & 1,38 \\
Lote grande & 2,61 & 1,27 \\
Produção em massa & 2,06 & 1,22 \\
\hline
\end{tabular}

Fonte: dados da pesquisa.

A aderência a parcerias, característica do outsourcing possibilita gerar eficácia interna no que tange a conhecimento e inovação e ao mesmo tempo requer um desenvolvimento contínuo dessas melhorias propiciando melhoras na oferta de produtos ou serviços das organizações. Os resultados dessa dimensão podem ser corroborados com estudos como Oshri, Kotlarsky e Gerbasi (2015) Moschuris (2008) evidenciam que a tecnologia de operações pode influenciar na decisão de comprar ou produzir, sendo considerada uma estratégia dinâmica e flexível em que a organização consegue mesclar tecnologias disponíveis no mercado com habilidades de produção.

Sob a dimensão da complexidade entende-se que quanto mais complexos são os produtos e serviços, maior é o número de contingências que pode afetar os resultados futuros das organizações, podendo este ser um cenário favorável para adesão ao outsourcing. As variáveis utilizadas para mensurar essa dimensão envolvem a complexidade técnica, o grau de diferenciação e a instalação especializada dos produtos e serviços, bem como a existência de técnicos de assistência pós-venda, apresentados nessa ordem de relevância pela amostra, conforme a Tabela 4.

Tabela 4 - Avaliação da dimensão da complexidade

\begin{tabular}{lll}
\hline Dimensão Complexidade & Média & Desvio Padrão \\
\hline Complexidade técnica & 3,45 & 1,27 \\
Grau de diferenciação & 3,03 & 1,21 \\
Instalação especializada & 2,88 & 1,40 \\
Existência de técnicos de assistência pós-venda & 2,64 & 1,36 \\
\hline
\end{tabular}

\section{Fonte: dados da pesquisa.}

Destaca-se que o item apontado como mais significativo na dimensão da complexidade é a complexidade técnica dos produtos, confirmando o estudo de Mclvor (2005) ao abordar que a prática de terceirar ou transferir à terceiros atividades da organização, envolve redesenho dos limites da organização e sua base de abastecimento. Isso gera conhecimento a respeito das próprias limitações se vendo diante da opção de aderir ao outsourcing.

Após esses achados, realizou-se a comparação de relevância das dimensões e os critérios utilizados para decisão de adesão ou não ao make or buy, apresentado na Tabela 5.

Tabela 5 - Comparação das dimensões

\begin{tabular}{lll}
\hline Dimensões & Média & Desvio Padrão \\
\hline Dimensão Complexidade & 3,00 & 1,06 \\
Dimensão dos Tipos de Tecnologias de Operações & 2,91 & 0,72 \\
Dimensões de Incerteza Ambiental & 2,61 & 1,02 \\
\hline
\end{tabular}

Fonte: dados da pesquisa. 
Pode-se observar que os gestores das industrias paranaenses participantes da amostra consideraram mais relevante, no momento decidir aderir à transferência de atividades ou serviços a terceiros, a dimensão da complexidade dos produtos e serviços ofertados, seguido pelos tipos de tecnologias utilizadas nas operações e a dimensão da incerteza ambiental é considerada por último nesse processo de tomada de decisão.

A pesquisa possibilitou identificar os elementos e variáveis considerados relevantes nesse processo de tomada de decisão pelos gestores respondentes e, embora não seja possível fazer uma generalização, os resultados fornecem uma indicação de como as empresas costumam resolver questões relacionadas ao dilema de produzir ou comprar.

\section{CONSIDERAÇÕES FINAIS}

O foco deste estudo foi investigar na percepção dos gestores, os critérios e as variáveis considerados relevantes para tomada de decisão de adesão ao outsourcing, o qual foi motivado pela pesquisa de Moschuris (2015). A relevância da pesquisa está na contribuição que o mesmo fornece ao agregar informações sobre a temática de outsourcing, uma vez que pesquisas sobre esse tema no Brasil ainda são escassas.

Os resultados obtidos evidenciaram que dentre os 11 critérios estratégicos abordados, os itens de custos e qualidade foram considerados como mais relevantes na percepção dos gestores respondentes para tomada de decisão de adesão ou não ao outsourcing, confirmando os achados de Moschuris (2015), entretanto, apresentando variações nas classificações de ordem de relevância dos demais itens.

Quanto as dimensões em que essas decisões são tomadas, a avaliada como mais importante pelos gestores, com base nas respostas dos questionários, foi a dimensão da complexidade, o que indica certa dificuldade por parte das empresas pesquisadas em relação a técnicas com maior complexidade na linha de produção, grau de diferenciação dos produtos ofertados e mão de obra especializada ante à opção de fabricação de produtos complexos e as pós vendas na organização.

As demais dimensões, tipos de tecnologias de operações e de incerteza ambiental, ocuparam o segundo e terceiro lugar respectivamente nas opiniões dos gestores como critérios recorrentes na decisão de produzir ou comprar, denotando-se que a dimensão de menor relevância é quanto a incerteza ambiental, enquanto que os tipos de tecnologia de operações é intermediária podendo ser justificada pela preocupação das organizações em garantir a inovação no processo produtivo e nos produtos ofertados, fator este que pode ser o diferencial para o aumento da participação no mercado e decisivo na decisão de 'make or buy'.

Os achados da pesquisa contribuem para a literatura contábil voltada ao tema em empresas paranaenses e o impacto das variáveis, como tipo e complexidade intrínsecas ao produto ou serviço e relativo a incerteza ambiental e a tecnologia disponível envolvendo questões táticas, justificando-se dessa forma a pesquisa, uma vez que o outsourcing difundese cada vez mais entre organizações sendo uma das decisões estratégicas que atraem o maior interesse de profissionais e estudiosos organizacionais.

Como limitação, enfatiza-se que os achados não podem ser generalizados por terem sido investigadas somente empresas do ramo industrial do estado do Paraná cadastradas na base de dados da FIEP do ano de 2012 que atuam com exportações e importações, bem como não obteve-se respostas suficientes para a aplicação de técnicas estatísticas inferenciais que possibilitassem a generalização dos achados. Para futuras pesquisas, recomenda-se uma abordagem maior 'de empresas de diferentes segmentos, bem como uma maior 
abrangência na questão territorial, buscando identificar os critérios aqui abordados em mais regiões do território nacional, ou mesmo fazendo comparações entre empresas de mesmo ramo em diferentes estados ou regiões. Recomenda-se também a utilização de um banco de dados mais atualizado que possibilite o enquadramento de empresas mais recentes, bem como a exclusão daquelas que já deixaram de operar.

\section{REFERÊNCIAS}

AUBERT, B. A.; KISHORE, R.; IRIYAMA, A. Exploring and managing the innovation through outsourcing paradox. The Journal of Strategic Information Systems, v. 24, n. 4, p. 255-269, 2015.

BAATARTOGTOKH, B.; DUNBAR, W. S.; VAN ZYL, D. The state of outsourcing in the Canadian mining industry. Resources Policy, v. 59, p. 184-191, 2018.

BAJEC, P.; JAKOMIN, I. A make-or-buy decision process for outsourcing. Promet-Traffic \& Transportation, v. 22, n. 4, p. 285-291, 2010.

BALS, L.; KIRCHOFF, J. F.; FOERSTL, K. Exploring the reshoring and insourcing decision making process: toward an agenda for future research. Operations Management Research, v. 9, n. 34, p. 102-116, 2016.

BALS, L.; TURKULAINEN, V. Achieving efficiency and effectiveness in Purchasing and Supply Management: Organization design and outsourcing. Journal of Purchasing and Supply Management, v. 23, n. 4, p. 256-267, 2017.

BENGTSSON, L.; VON HAARTMAN, R.; DABHILKAR, M. Low-cost versus innovation: contrasting outsourcing and integration strategies in manufacturing. Creativity and Innovation Management, v. 18, n. 1, p. 35-47, 2009.

BURT, D. N.; PETCAVAGE, S. D.; PINKERTON, R. L. Supply Management. International Edition ed. New York: McGraw Hill, 2010.

CARVALHO, J. M. M. Planeamento estratégico - O seu guia para o sucesso. Lisboa: Vida Econômica, v. 2, 2012.

COOPER, D. R.; SCHINDLER, P. S. Métodos de pesquisa em administração. 7. ed. Porto Alegre: Bookman, 2003.

EL MOKRINI, A. et al. An approach to risk Assessment for Outsourcing Logistics: Case of Pharmaceutical Industry. IFAC-PapersOnLine, 49(12), 1239-1244, 2016.

ESPINO-RODRÍGUEZ, T. F.; PADRÓN-ROBAINA, V. A review of outsourcing from the resourcebased view of the firm. International Journal of Management Reviews, v. 8, n. 1, p. 49-70, 2006.

FERRUZZI, A. M. et al. Reasons for outsourcing services in medium and large companies. Brazilian Business Review (English Edition), v. 8, n. 4, 2011.

FIGUEIREDO, R. M. C.; BREMER, C. F; MALDONADO, J. C. Evolução dos modelos de outsourcing: $\mathrm{O}$ estado da arte da literatura dos novos provedores de serviços de aplicativos. Perspectivas em Ciência da Informação, v. 8, n. 1, 2003.

GANZER, P. P. et al. Estratégias para a inovação: um estudo na RBS TV Caxias do Sul. Race: Revista de Administração, Contabilidade e Economia, v. 12, n. 1, p. 99-138, 2013. 
GERBL, M. et al. A multi-theory approach to understanding the business process outsourcing decision. Journal of World Business, v. 50, n. 3, p. 505-518, 2015.

GUIMARÃES, M. M. M. A. S. A importância das decisões make-or-buy nas atividades de Marketing de uma empresa: o caso do Continente. Dissertação (Mestrado em Economia) Faculdade de Economia do Porto. Porto, Portugal, 2017.

GUNASEKARAN, et al. Performance measures and metrics in outsourcing decisions: A review for research and applications. International Journal of Production Economics, v. 161, p. 153166, 2015.

KAKABADSE, A.; KAKABADSE, N. Outsourcing: current and future trends. Thunderbird International Business Review, v. 47, n. 2, p. 183-204, 2005.

KAMBLE, S. S.; GHOSH, S. Outsourcing printing services as a competitive manufacturing strategy: a case study. International Journal of Procurement Management, v. 3, n. 1, pp. 1231, 2010.

KULKARNI, S. V.; JENAMANI, M. Make-or-buy: a case study at an Indian automobile company. Strategic Outsourcing: An International Journal, v. 1, n. 3, p. 268-287, 2008.

LEE, G. et al. How manufacturers' long-term orientation toward suppliers influences outsourcing performance. Industrial Marketing Management, v. 74, p. 288-297, 2018.

LEEMAN, D.; REYNOLDS, D. Trust and outsourcing: Do perceptions of trust influence the retention of outsourcing providers in the hospitality industry? International Journal of Hospitality Management, v. 31, n.2, p. 601-608, 2012.

MAGELSSEN, C.; SANCHEZ, F.; DAMANPOUR, F. Learning from outsourcing: the effects of outsourcing strategy on organizational efficiency. In: Academy of Management Proceedings. Briarcliff Manor, NY 10510: Academy of Management, 2015. p. 17468.

MCIVOR, R. The Influence of Transaction Cost Economics and the Resource-based View on the Outsourcing Process. Sixteenth Annual Conference of POMS, Chicago, IL, April 29 - May 2, 2005.

MCIVOR, R. How the transaction cost and resource-based theories of the firm inform outsourcing evaluation. Journal of Operations Management, v. 27, p. 45-63, 2009.

MCIVOR, R. Understanding the Manufacturing Location Decision: The Case for the Transaction Cost and Capability Perspectives. Journal of Supply Chain Management, v. 49(2), p. 23-26, 2013.

MEIXELL, M.; KENYON, G.; WESTFALL, P. The effects of production outsourcing on factory cost performance: an empirical study. Journal of Manufacturing Technology Management, v. 25, n. 6, p. 750-774, 2014.

MOSCHURIS, S. J. Decision-making criteria in tactical make-or-buy issues: an empirical analysis. EuroMed Journal of Business, v. 10, n. 1, p. 2-20, 2015.

MOSCHURIS, S. J. Organizational participants in the make-or-buy process. Industrial Marketing Management, v. 37, n. 2, p. 143-153, 2008.

MOSCHURIS, S. J. Triggering mechanisms in make-or-buy decisions: An Empirical Analysis. Journal of Supply Chain Management, v. 43, n. 1, p. 40-49, 2007. 
MOURA Jr, P. J. Terceirização como estratégia de gestão do conhecimento. Cadernos EBAPE. BR, 15(2), 229-255, 2017.

NIELSEN, L. B.; MITCHELL, F.; NØRREKLIT, H. Management accounting and decision making: Two case studies of outsourcing. In: Accounting Forum. Elsevier, 2015. p. 64-82.

OSHRI, I.; KOTLARSKY, J.; GERBASI, A. Strategic innovation through outsourcing: the role of relational and contractual governance. The Journal of Strategic Information Systems, v. 24, n. 3, p. 203-216, 2015.

PRATAP, S. Towards a framework for performing outsourcing capability. Strategic Outsourcing: An International Journal, v. 7, n. 3, p. 226-252, 2014.

SAMPIERI, R. H.; COLLADO, C. F.; LUCIO, P. B. Metodologia de pesquisa. São Paulo: McGrawHill, 2013.

TAVANA, M.; ZAREINEJAD, M.; DI CAPRIO, D.; KAVIANI, M. A. An integrated intuitionistic fuzzy AHP and SWOT method for outsourcing reverse logistics. Applied Soft Computing, 40, 544557, 2016.

TROACĂ, V. A.; BODISLAV, D. A. Outsourcing. The Concept. Theoretical and Applied Economics, v. 6, n. 6, p. 51, 2012.

VERNALHA, H. B.; PIRES, S. R.I. Um modelo de condução do processo de outsourcing e um estudo de caso na indústria de processamento químico. Revista Produção, v. 15, n. 2, p. 273285, 2005.

WONG, C. W.; LAI, K.H; CHENG, T. C. E. Value of information integration to supply chain management: roles of internal and external contingencies. Journal of Management Information Systems, v. 28, n. 3, p. 161-200, 2011.

$\mathrm{ZHU}, \mathrm{X}$. Managing the risks of outsourcing: Time, quality and correlated costs. Transportation Research Part E: Logistics and Transportation Review, 90, 121. 